\title{
Management of Vascularity Compromised Limb in A Rural Hospital
}

\author{
Authors \\ Dr Ishtyak Ahmed Mir ${ }^{1}$, Dr Shamim Akhter ${ }^{2}$ \\ ${ }^{1}$ B-Grade Surgeon Specialist, ${ }^{2}$ Assistant Surgeon \\ Department of Surgery, Community Health Centre, Banihal, Jammu \& Kashmir, India \\ Corresponding Author \\ Dr Ishtyak Ahmed Mir \\ R/O, C-1, Old Medical enclave, Near K.C Cinema Jammu.180001 \\ Email: iamir327@yahoo.com, Phone No: 9419081019
}

\begin{abstract}
The study was conducted to know the presentation, symptomatology, clinical signs and management outcome of post traumatic painful and swollen limbs in a Community Health Centre. 21 patients managed for post traumatic edema and pain of the limb are presented. Males outnumbered females. Blunt trauma was the most common mode of injury, and lower limb was involved in majority. Majority were under treatment of quacks. Diagnosis of post traumatic swollen and vascular compromised limb (compartment syndrome) requiring some mode of treatment was made on clinical grounds, and pulse oxymetry. 10 patients underwent fasciotomy, 6 were managed conservatively and 4 patients underwent amputation. All the patients managed by conservative and surgical therapy had successful outcome.
\end{abstract}

Key Words: Fasciotomy, Gangrene, Splints, Trauma Limbs

\section{INTRODUCTION}

Post traumatic pain and edema limb a seemingly benign condition can present with a grim phenomenon if not managed by qualified professionals, because of squeal of trauma, or if mismanaged by professionals / quacks. Majority of these injuries may go unnoticed, but patients usually seek help from professional or quacks if they have pain and edema. In rural areas majority of these patients still go to quacks for management of such problems. Quacks who don't have any know how of the problem aggressively treat all such patients with one or the other methods, while doing so patients may at times not only loose their limb but life also.

Effusion or hemorrhage in to the tight osseofascial compartment of the fore arm, thigh and the leg cause increase in the inter compartmental pressure which may cause muscular ischemia and eventual muscle necrosis. Increasing numbers of vehicular accidents, assaults, conflicts, natural and man made disasters, fall, fire arm injuries, iatrogenic or in any other trauma limbs are invariably involved. Fractures and dislocations with associated vascular compromise especially in popliteal and cubital fossa if not managed on emergency basis and properly by a qualified professional may ultimately cause severe morbidity or mortality.

Pain, edema, restricted movements of a limb is compounded by mismanagement of a severe or even a trivial limb injury by quacks and inexperienced professionals, leading to compartment syndrome. The tragedy of majority of these victims attending quacks or getting tight 
splints is probably the common cause of compartment syndrome in rural areas. Since majority of these problems can be avoided and solved by simply removing the tight splints/ casts and if need be by performing fasciotomy, it is mandatory that such a seemingly benign condition be managed carefully. The study was conducted to know the mode / pattern of presentation of posttraumatic swollen / painful limb, assess the vascularity compromise, act promptly in case of vascularity compromise / tight compartment and devise ways and means in dealing with such patients in a community health centre, at the same time not forgetting that referral should not be delayed which may cost life or limb. If the patients report immediately, and there is no sign of transection of a major vessel, fasciotomy helps in relieving the symptoms and improving the vascularity. Pulse oxymetry is the lone helps to a surgeon in a rural centre where facilities for sophisticated investigatin are not available, and where patients refuse referral to higher centres.

\section{MATERIAL AND METHODS}

The study is conducted over a period of 4 years, in a community health center (CHC), the hospital is located on the national highway and besides elective, deals with all emergency cases round the clock. A detailed history, thorough general and systemic examination is recorded in all. Only patients with exclusively limb injuries, managed for limb injuries, initially by specialists, higher hospitals, or quacks are included in the study. Patients with compound fractures, major vascular / bony injuries, and associated other systemic injuries or illness, who directly came to over hospital, and all those patients (except those referred for amputation), who ultimately agreed for referral are not included in the study. Patients with limb injuries having mild pain, mild edema and who have no splint or plaster given any where, and also the patients who at removal of the splint have no evidence of edema, tenderness, or vascular compromise are not included in the study. Patients who have history of trauma, splints, intervention by professionals or quacks, pain / edema limb, painful movements of joints / limb, and who have clinical features of limb edema, decreased temperature compared to other limb, tenderness, restricted / painful movements, ischemia, anesthesia and gangrene are admitted.

A trial of conservative treatment is given to patients with,

1. Pain of the limb,

2. decreased temperature of the affected limb,

3. edema of the limb but no tight compartment,

4. edematous limb with feeble or absent pulses distally,

5. edematous limb with mild pain and no anesthesia,

6. pulse oxymetry evidence of equal or decreased saturation.

Conservative treatment consisted of

a) releasing the pressure support, splint / cast,

b) elevation of the limb,

c) antibiotics, analgesics, prophylactic proton pump inhibitors,

d) anticoagulants and antiplatelet, in some, if there was no contraindication,

e) physiotherapy is started after improvement in symptoms,

Surgical intervention (fasciotomy) is performed in patients

i. with failure to conservative treatment,

ii. edematous limb with severe pain,

iii. decreased or absent capillary and venous filling,

iv. pain on passive movements,

v. woody hard consistency of edematous part,

vi. early and progressive edema of the limb with tense compartment on palpation,

vii. edematous limb with colour and temperature changes,

viii. pulse oximetry showing decreased saturation. 
However, post traumatic and post splint woody hard consistency of edematous part, severe pain, colour/temperature changes, paraesthesia, pulselessness and decreased saturation on pulse oxymetry findings are taken as absolute indications of surgery, measures used in conservative treatment are continued after surgery also, besides fasciotomy is performed in only those patients who refused referral to higher and good centers.

Fasciotomy is performed by decompressing volar and dorsal compartment in fore arm, anterior, posterior and medial compartment in thigh, and anterior lateral, superficial posterior, and deep posterior in the leg, patients with fractures are given supports, slab to stabilize the limb. Post surgery patients are dressed on daily basis and results of management, morbidity and mortality are recorded.

\section{RESULTS}

21 patients with post traumatic history of pain and edema limb, had painful movements of joints / limb and intervention by quacks and or professionals. Clinical features included, limb edema, tenderness, restricted and painful movements, ischemia, anesthesia and gangrene. The patients included 17 males, a 14 year old boy and a 68 year old male. 13 patients were laborers; blunt trauma was the most common mode of injury. All the patients had either been managed by quacks or had been to quacks after being under treatment of specialists or at higher centers. Leg was the involved limb in 12 , thigh and leg in 2 , forearm in 4, whole arm in 2 and more then one limb was involved in one patient. Simple, in alignment, hair line fractures of bones as observed were, shaft of tibia 5 , upper end of tibia 2 , radius in 2, radius and ulna in 1.6 patients with fracture had initial treatment / stabilization done by specialists privately or at higher centers, they had left against medical advice and were managed at home or had gone to quacks, who had either changed or compounded the treatment line.

Tightly applied splints, wooden pressure supports, and splint related pressure symptoms were present clinically in all. 5 patients had clinical features of non salvageable limb at presentation (figure 1), these patients were initially managed by quacks, than some by specialists, again by quacks, and were trying to avoid amputation at any cost. These patients were managed for some time, during which period they were properly explained the chances of limb salvage at a higher center because of better facilities, and also the merits and demerits of amputation. One patient did not agree for referral and amputation, instead left against medical advice and died at home, 4 had amputation done.

Fasciotomy performed was lateral in 3, volar in 1, lateral and superficial posterior 2, anterior and deep posterior 2, medial 1, and volar and dorsal in 1 patient. Surgical interventions decompression, debridement in patients who had features of gangrene at admission and who were desperate to avoid amputation, was of no use, except for some psychological advantage to the patients who thought some limb saving exercise is going on. 11 patients, none of whom had any fractures were managed without any surgical intervention. The improvement in symptomatology was assessed by decrease in intensity of pain, improving temperature, increasing saturation, improving limb movements, progressive decrease in edema and tenderness. Fasciotomy wound was allowed to heal by primary intention in 5 , secondary intention in 3 and by delayed closure in 2 patients. Except for one, unnecessary very tight pressure supports / splints was the cause of compartment syndrome

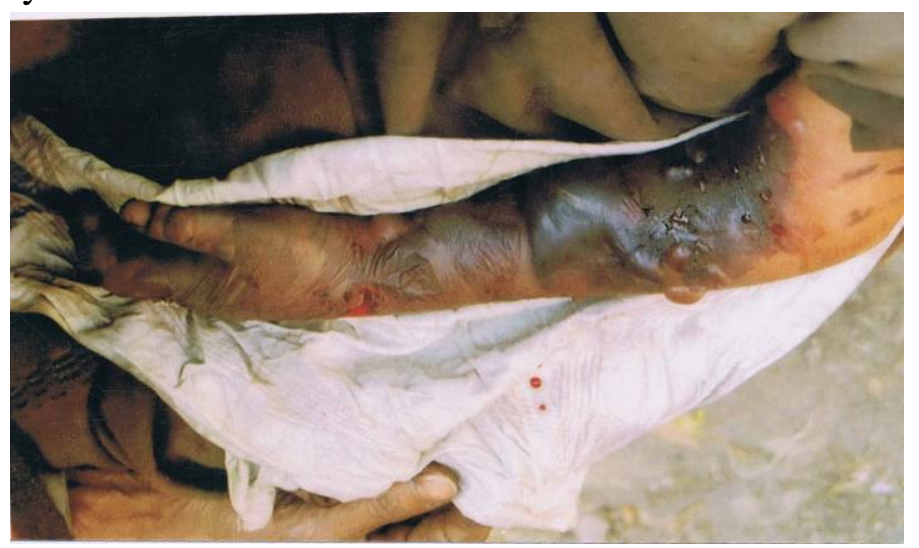

Figure 1 Photograph of a patient showing gangrenous changes in arm 


\section{COMMENTS}

Limb compartment problem has been recognized since $1881^{[1]}$, it was only in 1926 that the compartment problem was demonstrated experimentally $^{[2]}$. The first reported treatment of compartment syndrome appeared in $1888^{[3]}$. The initial injury causes increase in inter compartmental pressure, ultimately causing hypertension at the venous ends of the capillary beds, eventually arteriolar compression occurs leading to muscle infarction and nerve damage if patient is not treated ${ }^{[4-5]}$. Compartment syndrome may be because of orthopedic, vascular, soft tissue or iatrogenic injury. In the present study for lack of facilities, the diagnosis was made on clinical findings only. It could be that in some of the patients the condition was over estimated, also the duration of symptomatology could not be ascertained with certainty because of delayed presentation. The results of the present study that fracture tibia is common cause of compartment syndrome are in accordance to the results from other studies ${ }^{[6-8]}$. Fracture of radius and ulna causing compartment syndrome is well known ${ }^{[9-}$ 10]. Trauma being the common cause of compartment syndrome in present study, besides traumatic vascular injury, vascular procedures can result in compartment syndrome ${ }^{[11-12]}$, for lack of facilities it was not possible to assess the gravity of vascular injury, nor did we perform any vascular procedure, so it is not possible to draw a conclusion that vascular injury and vascular procedures were the cause of compartment in our study. The observations of the present study are in consistence to those of others who also noted that, soft tissue injury is the common cause of edematous limb, and pain may be due to direct blow to a muscle compartment, prolonged limb compression, and at times due to burns ${ }^{[13-14]}$.We did not observe any patient with iatrogenic injury, in iatrogenic causes intravenous and intra arterial injections can be the causative factor ${ }^{[15]}$. Anterior compartment being commonly effected in leg and flexor compartment in forearm is well known ${ }^{[16]}$. Single or double incision fasciotomy with or without fibular excision are commonly performed for leg .In the forearm volar faciotomy is the method of choice, but concurrent dorsal fasciotomy is also performed. Aggressive fasciotomies were not done in the present study because of non availability of pressure studies, and seemingly benign pathology clinically in majority, further whatever little was done that did help in achieving the goal.

At least in patients who had been managed by specialist initially, the morbidity could have been avoided or even reduced had these patients not left against specialist advice or had they not been mismanaged at home or by quacks. Even after splint, slab, or plaster given by a specialist, the quack removes such a support, in some breaks the fracture again and then uses his own pressure supports. It is a fact that quacks apply circumferential tight pressure supports not only in fractures but in sprains, simple soft tissue injuries, and even in patients with only history of trivial trauma. The problems get compounded because quacks have no idea of anatomy, type of injury, can't anticipate post pressure complications, and apply pressure equally to all injuries without even explaining the chances of pressure symptoms. It is because of lack of knowledge, ignorance, illiteracy, that patients choose quacks as the first line of treatment. Also specialist / professionals in good sense may explain these patients about the duration of treatment, levels of treatment, multispecialty involvement, and possibility of amputation, the fear of which makes them to leave the specialist / specialized hospital, and move towards quacks.

In summary majority of the problems were because of mismanagement, diagnosis was made on clinical grounds, those who came with established gangrene could not be helped, conservative treatment and fasciotomy done definitely helped in avoiding morbidity and mortality.

In conclusion ways and means should be devised in information, education and communication to make the public aware of preventable morbidity and mortality, quacks should be discouraged from managing such patients, every attempt should be 
made to refer these patients to qualified specialists or higher centres for better management, however, those who refuse referral should be managed aggressively besides conservative treatment, fasciotomy and debridement if indicated should not be delayed, to achieve the best results even in a $\mathrm{CHC}$,

\section{BIBLIOGRAPHY}

1. Volkmann R. Die ischaemischen Muskellahmungen and Kontrakturen. Centralbl chir $1881 ; 8:$ 801-3.

2. Jepson $\mathrm{P} N$. Ischemic contracture : experimental study. Ann Surg 1926 Dec; 84 (6): 785-95.

3. Rorabeck $\mathrm{CH}$. The treatment of compartment syndrome of the leg. J Bone Joint Surg Br 1984; 66: 93-7.

4. Lagerstrom CF, Reed RL II, Rowlands BJ, Fisher RP. Early fasciotomy for acute clinically evident posttraumatic compartment syndrome. Am J Surg, 1989 Jul; 158 (1): 36-9.

5. Mubarak SJ, Owen CA, Hargens AR, Garetto LP, Akeson WH. Acute compartment syndrome : diagnosis and treatment with the aid of a wick catheter. $\mathrm{J}$ Bone Joint Surg Am 1978; 60: 1091-5.

6. Turen $\mathrm{CH}$, Burgess AR, Vanco B. Skeletal stabilization for tibial fracture associated with acute compartment syndrome. Clin Orthop 1995 Jun; 315: 163-8.

7. McQueen MM, Gaston P, Court-Brown CM. Acute compartment syndrome, Who is at risk ? J Bone Joint Surg Br 2000 Mar; 82 (2): 200-3.

8. Mullet H, Al Abed K, Prasad CVR, O'Sullivan M. Outcome of compartment syndrome following intramedulary nailing of tibial diaphyseal fractures. Injury 2001; 32: 411-13.

9. Gilberman RH, Garfin SR, Hergenroeder PT, Mubarak SJ, Menon J. Compartment syndrome of the forearm: diagnosis and treatment. Clin Orthop 1981 Nov-Dec; 161: 252-61.

10. Royal SG. Compartment syndrome following forearm fracture in children. Injury 1990; 21: 73-6.

11. Ascer E, Strauch B, Calligaro KD, Gupta SK, Veith FJ. Ankle and foot fasciotomy: an adjunctive technique to optimize limb salvage after revascularization for acute ischemia. J Vasc Surg 1989 Apr; 9 (4): 594-7.

12. Hallidav AW, Taylor PR, Wolfe JH, Mansfield AO. The management of popliteal aneurysm: the importance of early surgical repair. Ann R Coll Surg Engl 1991 Jul; 73 (4): 253-7.

13. Schwartz JT Jr, Brumback RJ, Lakatos R, Poka A, Bathon GH, Burgess AR. Acute compartment syndrome of the thigh; A spectrum of injury. J bone Joint Surg Am 1989 Mar; 71 (3): 392-400.

14. Justis DL, Law EJ, MacMillan BG. Tibial compartment syndrome in burn patients, a report of four cases. Arch Surg 1976 Sep; 111 (9): 1004-8.

15. Ouellette EA, Kelly R. Compartment syndromes of the hand. J Bone Joint Surg Am 1996 Oct; 78 (10): 1515-22.

16. Sheridan GW, Matsen FA III. Fasciotomy in the treatment of acute compartment syndrome. J Bone Joint Surg Am 1976; 58-A: 112-15. 